\title{
Networked Control with a Stochastic Scheduling: a Time-Delay Approach *
}

\author{
Kun Liu ${ }^{*}$ Emilia Fridman ${ }^{* *}$ Karl Henrik Johansson * \\ * ACCESS Linnaeus Centre and School of Electrical Engineering, \\ KTH Royal Institute of Technology, SE-100 44 Stockholm, \\ Sweden(e-mails: kunliu; kallej@kth.se). \\ ** School of Electrical Engineering, Tel-Aviv University, 69978, \\ Tel-Aviv, Israel (e-mail: emilia@eng.tau.ac.il)
}

\begin{abstract}
This paper is concerned with the stability analysis of networked control systems with communication constraints, variable delays and variable sampling intervals. The scheduling of sensor communication is defined by a stochastic protocol. The activation probability of each sensor node is a given constant, whereas it is assumed that collisions occur with a certain probability. The resulting closed-loop system is a stochastic impulsive system with delays in the continuous dynamics and in the reset equations. The system matrices have stochastic parameters with Bernoulli distributions. Sufficient conditions for the exponential mean-square stability are derived via a Lyapunov-Krasovskii-based method. The efficiency of the method is illustrated on the examples of a cart-pendulum system and a batch reactor. It is demonstrated how our time-delay approach allows treating network-induced delays larger than the sampling intervals.
\end{abstract}

Keywords: networked control systems, Lyapunov functional, stochastic protocol, stochastic impulsive system

\section{INTRODUCTION}

Networked Control Systems (NCSs) are systems with the control loops closed over networks where sensors, controllers and actuators can be distributed over a wide geographical area (see e.g., Antsaklis and Baillieul [2007]). In many such systems, only one node is allowed to use the communication channel at once. The communication along the data channel is then orchestrated by a scheduling rule called protocol. Three recent approaches for NCSs are based on discrete-time systems (Donkers et al. [2011], Fujioka [2009]), impulsive/hybrid systems (Heemels et al. [2010], Nesic and Liberzon [2009]) and time-delay systems (Fridman et al. [2004], Fridman [2010], Gao and Chen [2008]).

The time-delay approach has been introduced recently for the stabilization of NCSs under the Round-Robin (RR) protocol in Liu et al. [2012b] and under a weighted Try-Once-Discard (TOD) protocol in Liu et al. [2012a]. The closed-loop system is modeled as a switched system with multiple and ordered time-varying delays under RR scheduling or as a hybrid system with time-varying delays in the dynamics and in the reset equations under the TOD scheduling. Differently from the hybrid and discrete-time approaches, the transmission delay is allowed to be larger than the sampling interval, but for a crucial point is that data packet dropout is not allowed for large delays in Liu et al. [2012b] and Liu et al. [2012a].

A stochastic protocol was introduced in Tabbara and Nesic [2008] and analyzed for the input-output stability of NCSs

* This work has been supported by the Knut and Alice Wallenberg Foundation and the Swedish Research Council. in the presence of data packet dropouts or collisions based on hybrid systems. An i.i.d (independent and identicallydistributed) sequence of Bernoulli random variables is applied to describe the stochastic protocol. Communication delays, however, are not included in the analysis. The stability of NCSs under a stochastic protocol, where the activated node is modeled by a Markov chain, has been studied in Donkers et al. [2012] by applying the discretetime modeling framework. In Donkers et al. [2012], data packet dropouts can be regarded as prolongations of the sampling interval for small delays.

In the present paper, to overcome the gap between the data packet dropouts phenomenon and the large communication delays for scheduling protocols (e.g., deterministic protocols: RR and TOD), we develop the timedelay approach to the stability analysis of NCSs under a stochastic protocol. As in Liu et al. [2012b] and Liu et al. [2012a], differently from the hybrid and discretetime approaches, we allow the transmission delays to be larger than the sampling intervals. For the simplicity of technical development, we consider a system with only two sensor nodes. A Remark 4 is given for extension to the case of more sensors nodes. The activation probability of each node is a given constant, whereas it is assumed that the collisions occur with a certain probability. The resulting closed-loop system is a stochastic impulsive system with delays in the continuous dynamics and in the reset equations. The system matrices have stochastic parameters with Bernoulli distributions. By developing appropriate Lyapunov-Krasovskii techniques, we derive Linear Matrix Inequalities (LMIs) conditions for the exponential meansquare stability of closed-loop system. The efficiency and 
advantages of the presented approach are illustrated by two examples.

Notation: Throughout the paper, the superscript ' $T$ ' stands for matrix transposition, $\mathcal{R}^{n}$ denotes the $n$ dimensional Euclidean space with vector norm $|\cdot|, \mathcal{R}^{n \times m}$ is the set of all $n \times m$ real matrices, and the notation $P>0$, for $P \in \mathcal{R}^{n \times n}$ means that $P$ is symmetric and positive definite. The symmetric elements of the symmetric matrix will be denoted by $*$. The space of functions $\phi:\left[-\tau_{M}, 0\right] \rightarrow$ $\mathcal{R}^{n}$, which are absolutely continuous on $\left[-\tau_{M}, 0\right]$, and have square integrable first-order derivatives is denoted by $W\left[-\tau_{M}, 0\right]$ with the norm $\|\phi\|_{W}=\max _{\theta \in\left[-\tau_{M}, 0\right]}|\phi(\theta)|+$ $\left[\int_{-\tau_{M}}^{0}|\dot{\phi}(s)|^{2} d s\right]^{\frac{1}{2}} \cdot \mathbb{Z}$ denotes the set of non-negative integers.

\section{SYSTEM MODEL}

\subsection{NCS model}

Consider the system architecture in Fig.1 with plant

$$
\dot{x}(t)=A x(t)+B u(t),
$$

where $x(t) \in \mathcal{R}^{n}$ is the state vector, $u(t) \in \mathcal{R}^{m}$ the control input and $A, B$ are system matrices of appropriate dimensions. The initial condition is given by $x(0)=x_{0}$.

The NCS has two sensors, a controller and an actuator connected via two networks. For the sake of simplicity, we limit the presentation to two sensor nodes. Their measurements are given by $y_{i}(t)=C_{i} x(t), i=1,2$. Let $C=\left[\begin{array}{l}C_{1} \\ C_{2}\end{array}\right], y(t)=\left[\begin{array}{l}y_{1}(t) \\ y_{2}(t)\end{array}\right] \in \mathcal{R}^{n_{y}}$. We denote by $s_{k}$ the unbounded and monotonously increasing sequence of sampling instants

$$
0=s_{0}<s_{1}<\ldots<s_{k}<\ldots, \quad k \in \mathbb{Z}, \lim _{k \rightarrow \infty} s_{k}=\infty .
$$

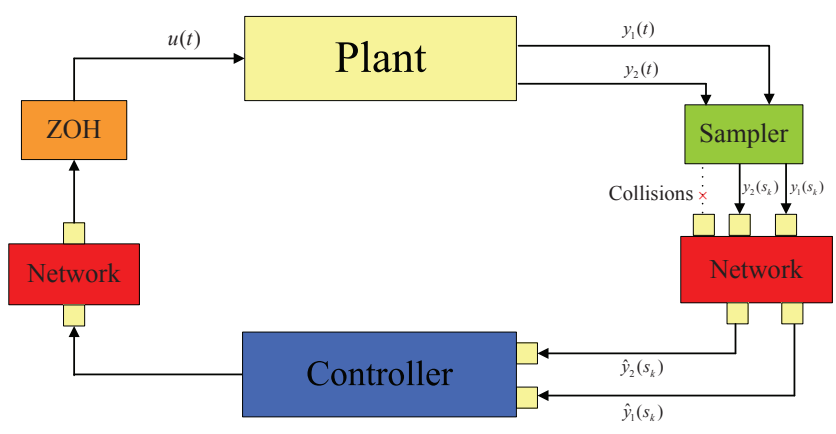

Fig. 1. NCS architecture

At each sampling instant $s_{k}$, at most one of the outputs $y_{i}\left(s_{k}\right) \in \mathcal{R}^{n_{i}}\left(n_{1}+n_{2}=n_{y}\right)$ is transmitted over the network. We suppose that the transmissions over the two networks are subject to a variable total delay $\eta_{k}=\eta_{k}^{s c}+$ $\eta_{k}^{c a}$, where $\eta_{k}^{s c}$ and $\eta_{k}^{c a}$ are the network-induced delays from the sensor to the controller and from the controller to the actuator, respectively. Then $t_{k}=s_{k}+\eta_{k}$ is the updating time instant of the Zero-Order Hold $(\mathrm{ZOH})$ device.

Differently from Donkers et al. [2011], Heemels et al. [2010], we do not restrict the network delays to be small.
Following Liu et al. [2012a] and Liu et al. [2012b], we allow the delay to be large provided that the previous sample cannot reach the destination (to the controller or to the actuator) after the current one. Assume that the networkinduced delay $\eta_{k}$ and the time span between the next updating instant $t_{k+1}$ and the current sampling instant $s_{k}$ are bounded:

$$
t_{k+1}-t_{k}+\eta_{k} \leq \tau_{M}, 0 \leq \eta_{m} \leq \eta_{k} \leq \eta_{M}, k \in \mathbb{Z}
$$

where $\tau_{M}$ denotes the maximum time span between the time $s_{k}=t_{k}-\eta_{k}$ at which the state is sampled and the time $t_{k+1}$ at which the next update arrives at the destination. Here $\eta_{m}$ and $\eta_{M}$ are known bounds and $\tau_{M}=M A T I+\eta_{M}$, where $M A T I$ denotes the Maximum Allowable Transmission Interval. Since $M A T I=\tau_{M}-$ $\eta_{M} \leq \tau_{M}-\eta_{m}, \eta_{m}>\frac{\tau_{M}}{2}$ implies that the network delays are non-small due to $\eta_{k} \geq \eta_{m}>\tau_{M}-\eta_{m}$. For the given examples in Section 4, we will show that when $\eta_{m}>\frac{\tau_{M}}{2}$ our method is feasible.

Remark 1. Differently from Naghshtabrizi et al. [2010], where subscript $k$ in $t_{k}$ corresponds to the measurements that are not lost, in our paper $k$ corresponds to the sampling time. This is because we consider the probability of collisions (see further details below).

Remark 2. We follow a commonly used assumption on the boundedness of the network-induced delays, e.g., Donkers et al. [2011], Gao and Chen [2008]. Another possibility is the Markov chain model of the network-induced delays in NCSs e.g., Huang and Nguang [2008], Nilsson [1998].

\subsection{Stochastic scheduling protocol}

Denote by $\hat{y}\left(s_{k}\right)=\left[\begin{array}{l}\hat{y}_{1}\left(s_{k}\right) \\ \hat{y}_{2}\left(s_{k}\right)\end{array}\right] \in \mathcal{R}^{n_{y}}$ the sensor output submitted to the scheduling protocol. At each sampling instant at most one of the system nodes $i \in\{1,2\}$ is active, that is at most one $\hat{y}_{i}\left(s_{k}\right)$ is updated with the recent output $y_{i}\left(s_{k}\right)$. The choice of the active output node is defined by a stochastic scheduling protocol. In some cases, collisions may occur when nodes access the network. If this happens, then packet with sensor data is dropped. The process $\hat{y}\left(s_{k}\right)$ is assumed to be i.i.d with the probabilities given by

$$
\begin{aligned}
& \operatorname{Prob}\left\{\hat{y}\left(s_{k}\right)=\left[\begin{array}{l}
\hat{y}_{1}\left(s_{k-1}\right) \\
\hat{y}_{2}\left(s_{k-1}\right)
\end{array}\right]\right\}=\beta_{0}, \\
& \operatorname{Prob}\left\{\hat{y}\left(s_{k}\right)=\left[\begin{array}{l}
y_{1}\left(s_{k}\right) \\
\hat{y}_{2}\left(s_{k-1}\right)
\end{array}\right]\right\}=\beta_{1}, \\
& \operatorname{Prob}\left\{\hat{y}\left(s_{k}\right)=\left[\begin{array}{l}
\hat{y}_{1}\left(s_{k-1}\right) \\
y_{2}\left(s_{k}\right)
\end{array}\right]\right\}=\beta_{2},
\end{aligned}
$$

where $\beta_{i}, i=0,1,2$ are positive scalars and $\sum_{i=0}^{2} \beta_{i}=1$.

\subsection{Stochastic impulsive time-delay model}

Following Liu et al. [2012a], we consider the error between the system output $y\left(s_{k}\right)$ and the last available information $\hat{y}\left(s_{k-1}\right)$ :

$$
\begin{aligned}
& e(t)=\operatorname{col}\left\{e_{1}(t), e_{2}(t)\right\} \equiv \hat{y}\left(s_{k-1}\right)-y\left(s_{k}\right), \\
& \quad t \in\left[t_{k}, t_{k+1}\right), k \in \mathbb{Z}, \hat{y}\left(s_{-1}\right) \triangleq 0, e(t) \in \mathcal{R}^{n_{y}} .
\end{aligned}
$$


We suppose that the controller and the actuator are eventdriven. $\hat{y}\left(s_{k}\right)=\left[\begin{array}{l}\hat{y}_{1}\left(s_{k}\right) \\ \hat{y}_{2}\left(s_{k}\right)\end{array}\right] \in \mathcal{R}^{n_{y}}(k \in \mathbb{Z})$ is the current output information at the controller.

Static output feedback control Assume that there exists a matrix $K=\left[\begin{array}{ll}K_{1} & K_{2}\end{array}\right], K_{1} \in \mathcal{R}^{m \times n_{1}}, K_{2} \in \mathcal{R}^{m \times n_{2}}$ such that $A+B K C$ is Hurwitz. The static output feedback controller has the form

$$
u(t)=u_{k}=K \hat{y}\left(t_{k}-\eta_{k}\right), \quad t \in\left[t_{k}, t_{k+1}\right), k \in \mathbb{Z} .
$$

Thus, (3), (4) and (5) are equivalent to

$$
\begin{gathered}
\operatorname{Prob}\left\{u_{k}=K_{1} \hat{y}_{1}\left(s_{k-1}\right)+K_{2} \hat{y}_{2}\left(s_{k-1}\right) \triangleq u^{0}\right\}=\beta_{0}, \\
\operatorname{Prob}\left\{u_{k}=K_{1} y_{1}\left(s_{k}\right)+K_{2} \hat{y}_{2}\left(s_{k-1}\right) \triangleq u^{1}\right\}=\beta_{1}, \\
\operatorname{Prob}\left\{u_{k}=K_{1} \hat{y}_{1}\left(s_{k-1}\right)+K_{2} y_{2}\left(s_{k}\right) \triangleq u^{2}\right\}=\beta_{2},
\end{gathered}
$$

respectively. Following Yue et al. [2009], we introduce the indicator functions

$$
\pi_{\left\{u_{k}=u^{i}\right\}}=\left\{\begin{array}{l}
1, u_{k}=u^{i} \\
0, u_{k} \neq u^{i},
\end{array} \quad i=0,1,2, k \in \mathbb{Z} .\right.
$$

Thus, we have

$$
\begin{aligned}
& \mathbb{E}\left\{\pi_{\left\{u_{k}=u^{i}\right\}}\right\}=\mathbb{E}\left\{\left[\pi_{\left\{u_{k}=u^{i}\right\}}\right]^{2}\right\}=\operatorname{Prob}\left\{u_{k}=u^{i}\right\}=\beta_{i}, \\
& \mathbb{E}\left\{\left[\pi_{\left\{u_{k}=u^{i}\right\}}-\beta_{i}\right]\left[\pi_{\left\{u_{k}=u^{j}\right\}}-\beta_{j}\right]\right\}=\left\{\begin{array}{l}
-\beta_{i} \beta_{j}, i \neq j, \\
\beta_{i}\left(1-\beta_{i}\right), i=j .
\end{array}\right.
\end{aligned}
$$

Therefore, we obtain the stochastic impulsive closed-loop system:

$$
\begin{aligned}
& \dot{x}(t)=A x(t)+A_{1} x\left(t_{k}-\eta_{k}\right)+\sum_{i=1}^{2}\left(1-\pi_{\left\{u_{k}=u^{i}\right\}}\right) B_{i} e_{i}(t), \\
& \dot{e}(t)=0, \quad t \in\left[t_{k}, t_{k+1}\right)
\end{aligned}
$$

where $A_{1}=B K C, B_{i}=B K_{i}, i=1,2$.

Next, taking into account (4), (5) and (11), we obtain

$$
\begin{aligned}
\hat{y}\left(s_{k}\right) & =\left[\begin{array}{l}
\hat{y}_{1}\left(s_{k}\right) \\
\hat{y}_{2}\left(s_{k}\right)
\end{array}\right] \\
& =\left[\begin{array}{l}
\pi_{\left\{u_{k}=u^{1}\right\}} y_{1}\left(s_{k}\right)+\left(1-\pi_{\left\{u_{k}=u^{1}\right\}}\right) \hat{y}_{1}\left(s_{k-1}\right) \\
\pi_{\left\{u_{k}=u^{2}\right\}} y_{2}\left(s_{k}\right)+\left(1-\pi_{\left\{u_{k}=u^{2}\right\}}\right) \hat{y}_{2}\left(s_{k-1}\right)
\end{array}\right] \\
& =y\left(s_{k}\right)+\left[\begin{array}{l}
\left(1-\pi_{\left\{u_{k}=u^{1}\right\}}\right) e_{1}\left(t_{k}\right) \\
\left(1-\pi_{\left\{u_{k}=u^{2}\right\}}\right) e_{2}\left(t_{k}\right)
\end{array}\right]
\end{aligned}
$$

which gives

$$
\begin{aligned}
e\left(t_{k+1}\right)=\hat{y}\left(s_{k}\right)-y\left(s_{k+1}\right) & \\
= & {\left[\begin{array}{c}
\left(1-\pi_{\left\{u_{k}=u^{1}\right\}}\right) e_{1}\left(t_{k+1}^{-}\right) \\
\left(1-\pi_{\left\{u_{k}=u^{2}\right\}}\right) e_{2}\left(t_{k+1}^{-}\right)
\end{array}\right]+y\left(s_{k}\right)-y\left(s_{k+1}\right) . }
\end{aligned}
$$

Thus, the delayed reset system is given by

$$
\begin{aligned}
x\left(t_{k+1}\right)= & x\left(t_{k+1}^{-}\right), \\
e\left(t_{k+1}\right)= & {\left[\begin{array}{c}
\left(1-\pi_{\left\{u_{k}=u^{1}\right\}}\right) e_{1}\left(t_{k+1}^{-}\right) \\
\left(1-\pi_{\left\{u_{k}=u^{2}\right\}}\right) e_{2}\left(t_{k+1}^{-}\right)
\end{array}\right] } \\
& +C x\left(t_{k}-\eta_{k}\right)-C x\left(t_{k+1}-\eta_{k+1}\right) .
\end{aligned}
$$

Since $x\left(t_{k}-\eta_{k}\right)=x(t-\tau(t))$ for $t \in\left[t_{k}, t_{k+1}\right)$ with $\tau(t)=t-t_{k}+\eta_{k} \in\left[\eta_{m}, \tau_{M}\right]$ (cf. (2)), the impulsive system model (13)-(14) contains the piecewise-continuous delay $\tau(t)$ in the continuous-time dynamics (13). Even for $\eta_{k}=0$, we have the delayed state $x\left(t_{k}\right)=x(t-$ $\tau(t))$ with $\tau(t)=t-t_{k}$. The initial condition for (13)(14) has the form of $x(t)=\phi(t), t \in\left[t_{0}-\tau_{M}, t_{0}\right]$ and $e\left(t_{0}\right)=-C x\left(t_{0}-\eta_{0}\right)=-C x_{0}$, where $\phi(t)$ is a continuous function on $\left[t_{0}-\tau_{M}, t_{0}\right]$.

Remark 3. Applying the Bernoulli-distributed stochastic variable $\pi_{\left\{u_{k}=u^{i}\right\}}, i=0,1,2$, the closed-loop system is given by an impulsive time-delay system with stochastic parameters in the system matrices. Note that the Bernoulli distribution has been applied to NCS with probabilistic measurements missing (Wang et al. [2006]), random packet losses (Wang et al. [2007]), stochastic sampling intervals (Gao et al. [2009]) and time-delay system with stochastic interval delays (Yue et al. [2009]).

Remark 4. Our results can be extended to the case of any $N \geq 3$ sensor nodes $y_{i}(t)=C_{i} x(t) \in \mathcal{R}^{n_{i}}, i=$ $1, \ldots, N, \sum_{i=1}^{N} n_{i}=n_{y}$. We denote $\tilde{C}^{T}=\left[C_{1}^{T} \ldots C_{N}^{T}\right]$, $\tilde{K}=\left[K_{1} \ldots K_{N}\right]$ and $\tilde{e}(t)=\operatorname{col}\left\{e_{1}(t), \ldots, e_{N}(t)\right\} \equiv$ $\hat{y}\left(s_{k-1}\right)-y\left(s_{k}\right), t \in\left[t_{k}, t_{k+1}\right), k \in \mathbb{Z}$. At each sampling instant $s_{k}$, the probabilities that $\hat{y}_{i}\left(s_{k}\right)$ is updated with the recent output $y_{i}\left(s_{k}\right)$ and collisions happen are denoted by $\beta_{i}>0, i=1, \ldots, N$ and $\beta_{0}>0$, respectively, where $\sum_{i=0}^{N} \beta_{i}=1$. Then the static output feedback controller has the form

$$
\begin{aligned}
u(t)= & u_{k}=K \hat{y}\left(t_{k}-\eta_{k}\right) \\
& =\left\{\begin{array}{l}
\sum_{j=1}^{N} K_{j} \hat{y}_{j}\left(s_{k-1}\right) \triangleq u^{0}, \\
\sum_{\substack{j=1, j \neq i \\
i=1, \ldots, N}}^{N} K_{j} \hat{y}_{j}\left(s_{k-1}\right)+K_{i} y_{i}\left(s_{k}\right) \triangleq u^{i},
\end{array}\right.
\end{aligned}
$$

with $\operatorname{Prob}\left\{u_{k}=u^{i}\right\}=\beta_{i}, i=0,1, \ldots, N$. Thus, the continuous dynamics of the stochastic impulsive closedloop model (1), (15) with $N$ sensor nodes can be expressed as

$$
\begin{aligned}
& \dot{x}(t)=A x(t)+\tilde{A}_{1} x\left(t_{k}-\eta_{k}\right)+\sum_{i=1}^{N}\left(1-\tilde{\pi}_{\left\{u_{k}=u^{i}\right\}}\right) \tilde{B}_{i} \tilde{e}_{i}(t), \\
& \dot{\tilde{e}}(t)=0, \quad t \in\left[t_{k}, t_{k+1}\right)
\end{aligned}
$$

where $\tilde{A}_{1}=B \tilde{K} \tilde{C}, \quad \tilde{B}_{i}=B \tilde{K}_{i}, \quad i=1, \ldots, N$ and $\tilde{\pi}_{\left\{u_{k}=u^{i}\right\}}=\left\{\begin{array}{l}1, u_{k}=u^{i} \\ 0, u_{k} \neq u^{i},\end{array} \quad i=0,1, \ldots, N, k \in \mathbb{Z}\right.$. The delayed reset system is given by

$$
\begin{aligned}
x\left(t_{k+1}\right)= & x\left(t_{k+1}^{-}\right), \\
\tilde{e}\left(t_{k+1}\right)= & {\left[\begin{array}{c}
\left(1-\tilde{\pi}_{\left\{u_{k}=u^{1}\right\}}\right) e_{1}\left(t_{k+1}^{-}\right) \\
\vdots \\
\left(1-\tilde{\pi}_{\left\{u_{k}=u^{i}\right\}}\right) e_{i}\left(t_{k+1}^{-}\right) \\
\vdots \\
\left(1-\tilde{\pi}_{\left\{u_{k}=u^{N}\right\}}\right) e_{N}\left(t_{k+1}^{-}\right)
\end{array}\right] } \\
& +\tilde{C} x\left(t_{k}-\eta_{k}\right)-\tilde{C} x\left(t_{k+1}-\eta_{k+1}\right) .
\end{aligned}
$$

Dynamic output feedback We consider the dynamic output feedback of (1) under the assumption that the controller is directly connected to the actuator, i.e., $\eta_{k}=\eta_{k}^{s c}$. The controller is assumed to be given by

$$
\begin{aligned}
& \dot{x}_{c}(t)=A_{c} x_{c}(t)+B_{c} \hat{y}\left(s_{k}\right), \\
& u(t)=C_{c} x_{c}(t)+D_{c} \hat{y}\left(s_{k}\right), \quad t \in\left[t_{k}, t_{k+1}\right), k \in \mathbb{Z},
\end{aligned}
$$


where $x_{c}(t) \in \mathcal{R}^{n_{c}}$ is the state of the controller, $A_{c}, B_{c}, C_{c}$ and $D_{c}$ are matrices of appropriate dimensions. The closed-loop system (1), (16) with (3)-(5) can be presented in the form of $(13),(14)$, where $e_{i}(t)(i=1,2)$ are given by $(6)$, the system state and matrices are replaced by the ones with bars as follows:

$$
\begin{gathered}
\bar{x}=\left[\begin{array}{cc}
x^{T} & x_{c}^{T}
\end{array}\right]^{T}, \quad \bar{A}=\left[\begin{array}{cc}
A & B C_{c} \\
0 & A_{c}
\end{array}\right], \bar{A}_{1}=\left[\begin{array}{c}
B D_{c}\left[\begin{array}{c}
C_{1} \\
C_{2}
\end{array}\right] \\
B_{c}\left[\begin{array}{l}
C_{1} \\
C_{2}
\end{array}\right]
\end{array}\right], \\
\bar{C}=\left[\begin{array}{l}
\bar{C}_{1} \\
\bar{C}_{2}
\end{array}\right]=\left[\begin{array}{cc}
C_{1} & 0 \\
0 & 0 \\
\hline 0 & 0 \\
C_{2} & 0
\end{array}\right], \bar{B}_{1}=\bar{B}_{2}=\left[\begin{array}{c}
B D_{c} \\
B_{c}
\end{array}\right], \\
\bar{e}(t)=\left[\begin{array}{c}
\bar{e}_{1}(t) \\
\bar{e}_{2}(t)
\end{array}\right], \bar{e}_{1}(t)=\left[\begin{array}{c}
e_{1}(t) \\
0
\end{array}\right], \bar{e}_{2}(t)=\left[\begin{array}{c}
0 \\
e_{2}(t)
\end{array}\right] .
\end{gathered}
$$

\section{EXPONENTIAL MEAN-SQUARE STABILITY OF STOCHASTIC IMPULSIVE DELAYED SYSTEM}

Definition 1. Impulsive system (13)-(14) is said to be exponentially mean-square stable with respect to $x$ if there exist constants $b>0, \kappa>0$ such that

$$
\mathbb{E}\left\{|x(t)|^{2}\right\} \leq b e^{-2 \kappa\left(t-t_{0}\right)} \mathbb{E}\left\{\left\|x_{t_{0}}\right\|_{W}^{2}+\left|e\left(t_{0}\right)\right|^{2}\right\}, t \geq t_{0}
$$

for the solutions of the stochastic impulsive system (13)(14) initialized with $e\left(t_{0}\right) \in \mathcal{R}^{n_{y}}$ and $x(t)=\phi(t), t \in\left[t_{0}-\right.$ $\left.\tau_{M}, t_{0}\right]$.

Our objective of this section is to derive LMI conditions for the exponential mean-square stability of the impulsive system (13)-(14) with respect to $x$. We extend the Lyapunov-Krasovskii Functional (LKF) introduced in Liu et al. [2012a] to the following form:

$$
\begin{aligned}
& V_{e}(t)=V\left(t, x_{t}, \dot{x}_{t}\right)+\sum_{i=1}^{2}\left(1-\beta_{i}\right) e_{i}^{T}(t) Q_{i} e_{i}(t), \\
& V\left(t, x_{t}, \dot{x}_{t}\right)=\tilde{V}\left(t, x_{t}, \dot{x}_{t}\right) \\
& +\left(\tau_{M}-\eta_{m}\right) \int_{t_{k}-\eta_{k}}^{t} e^{2 \alpha(s-t)} \dot{x}^{T}(s) Q \dot{x}(s) d s, \\
& \tilde{V}\left(t, x_{t}, \dot{x}_{t}\right)=x^{T}(t) P x(t) \\
& +\int_{t-\eta_{m}}^{t} e^{2 \alpha(s-t)} x^{T}(s) S_{0} x(s) d s \\
& +\int_{t-\tau_{M}}^{t-\eta_{m}} e^{2 \alpha(s-t)} x^{T}(s) S_{1} x(s) d s \\
& +\eta_{m} \int_{-\eta_{m}}^{0} \int_{t+\theta}^{t} e^{2 \alpha(s-t)} \dot{x}^{T}(s) R_{0} \dot{x}(s) d s d \theta \\
& +\left(\tau_{M}-\eta_{m}\right) \int_{-\tau_{M}}^{-\eta_{m}} \int_{t+\theta}^{t} e^{2 \alpha(s-t)} \dot{x}^{T}(s) R_{1} \dot{x}(s) d s d \theta, \\
& P>0, S_{i}>0, R_{i}>0, Q>0, Q_{j}>0, \alpha>0, \\
& i=0,1, j=1,2, t \in\left[t_{k}, t_{k+1}\right), k \in \mathbb{Z} \text {, }
\end{aligned}
$$

where $x_{t}(\theta) \triangleq x(t+\theta), \theta \in\left[-\tau_{M}, 0\right]$. Here the term

$$
e_{i}^{T}(t) Q_{i} e_{i}(t) \equiv e_{i}^{T}\left(t_{k}\right) Q_{i} e_{i}\left(t_{k}\right), t \in\left[t_{k}, t_{k+1}\right), i=1,2
$$

is piecewise-constant. The term $\tilde{V}\left(t, x_{t}, \dot{x}_{t}\right)$ represents the standard Lyapunov functional for systems with a time-varying delay $\tau(t) \in\left[\eta_{m}, \tau_{M}\right]$. The term $\left(\tau_{M}-\right.$ $\left.\eta_{m}\right) \int_{t_{k}-\eta_{k}}^{t} e^{2 \alpha(s-t)} \dot{x}^{T}(s) Q \dot{x}(s) d s$ is borrowed from Liu et al. [2012a] and is inserted to cope with the delays in the reset conditions. It is continuous on $\left[t_{k}, t_{k+1}\right)$ and does not grow at the jumps $t=t_{k+1}$, since

$$
\begin{aligned}
& \left(\tau_{M}-\eta_{m}\right) \mathbb{E}\left\{\int_{t_{k+1}-\eta_{k+1}}^{t_{k+1}} e^{2 \alpha\left(s-t_{k+1}\right)}|\sqrt{Q} \dot{x}(s)|^{2} d s\right\} \\
& -\left(\tau_{M}-\eta_{m}\right) \mathbb{E}\left\{\int_{t_{k}-\eta_{k}}^{t_{k+1}^{-}} e^{2 \alpha\left(s-t_{k+1}\right)}|\sqrt{Q} \dot{x}(s)|^{2} d s\right\} \\
& \leq-\left(\tau_{M}-\eta_{m}\right) e^{-2 \alpha \tau_{M}} \mathbb{E}\left\{\int_{t_{k}-\eta_{k}}^{t_{k+1}-\eta_{k+1}}|\sqrt{Q} \dot{x}(s)|^{2} d s\right\} \\
& \leq-e^{-2 \alpha \tau_{M}} \mathbb{E}\left\{\left|\sqrt{Q}\left[x\left(t_{k}-\eta_{k}\right)-x\left(t_{k+1}-\eta_{k+1}\right)\right]\right|^{2}\right\},
\end{aligned}
$$

where we applied Jensen's inequality (Gu et al. [2003]). The infinitesimal operator $\mathcal{L}$ of $V_{e}(t)$ is defined as

$$
\mathcal{L} V_{e}(t)=\lim _{\Delta \rightarrow 0^{+}} \frac{1}{\Delta}\left\{\mathbb{E}\left\{V_{e}(t+\Delta) \mid t\right\}-V_{e}(t)\right\} .
$$

The following lemma gives sufficient conditions for the exponential stability of (13)-(14) with respect to $x$ in the mean-square sense:

Lemma 1. If there exist positive constant $\alpha$, matrices $U_{i}>$ $0, i=1,2$ and $V_{e}(t)$ of (17) such that along (13)-(14) for $t \in\left[t_{k}, t_{k+1}\right)$

$$
\mathbb{E}\left\{\mathcal{L} V_{e}(t)+2 \alpha V_{e}(t)-\sum_{i=1}^{2} \frac{1-\beta_{i}}{\tau_{M}-\eta_{m}} e_{i}^{T}(t)\left(Q_{i}-U_{i}\right) e_{i}(t)\right\} \leq 0,
$$

with

$$
\Omega=\left[\begin{array}{ccc}
\Omega_{11} & \left(\beta_{0}+\beta_{2}\right)^{2} C_{1}^{T} Q_{1} & \left(\beta_{0}+\beta_{1}\right)^{2} C_{2}^{T} Q_{2} \\
* & \Omega_{22} & 0 \\
* & * & \Omega_{33}
\end{array}\right] \leq 0
$$

holds, where

$$
\begin{aligned}
& \Omega_{11}=\sum_{i=1}^{2}\left(1-\beta_{i}\right) C_{i}^{T} Q_{i} C_{i}-Q e^{-2 \alpha \tau_{M}}, \\
& \Omega_{22}=\left(\beta_{0}+\beta_{2}\right)^{2} Q_{1}-\left(\beta_{0}+\beta_{2}\right) U_{1}, \\
& \Omega_{33}=\left(\beta_{0}+\beta_{1}\right)^{2} Q_{2}-\left(\beta_{0}+\beta_{1}\right) U_{2} .
\end{aligned}
$$

Then $V_{e}(t)$ satisfies

$$
\mathbb{E}\left\{V_{e}\left(t_{k+1}\right)-V_{e}\left(t_{k+1}^{-}\right)+\sum_{i=1}^{2}\left(1-\beta_{i}\right) e_{i}^{T}\left(t_{k}\right)\left(Q_{i}-U_{i}\right) e_{i}\left(t_{k}\right)\right\} \leq 0 .
$$

Moreover, the following bound is valid for the solution of (13)-(14) with the initial condition $x_{t_{0}}, e\left(t_{0}\right)$ :

$$
\begin{aligned}
& \mathbb{E}\left\{V\left(t, x_{t}, \dot{x}_{t}\right)\right\} \leq e^{-2 \alpha\left(t-t_{0}\right)} \mathbb{E}\left\{V_{e}\left(t_{0}\right)\right\}, t \geq t_{0}, \\
& V_{e}\left(t_{0}\right)=V\left(t_{0}, x_{t_{0}}, \dot{x}_{t_{0}}\right)+\sum_{i=1}^{2}\left(1-\beta_{i}\right) e_{i}^{T}\left(t_{0}\right) Q_{i} e_{i}\left(t_{0}\right),
\end{aligned}
$$

implying the exponential mean-square stability of (13)(14) with respect to $x$.

Proof: From (18), we have for $t \in\left[t_{k}, t_{k+1}\right)$

$$
V_{e}(t)=V\left(t, x_{t}, \dot{x}_{t}\right)+\sum_{i=1}^{2}\left(1-\beta_{i}\right) e_{i}^{T}\left(t_{k}\right) Q_{i} e_{i}\left(t_{k}\right) .
$$


Since

$$
\int_{t_{k}}^{t} e^{-2 \alpha(t-s)} d s \leq \tau_{M}-\eta_{m}, t \in\left[t_{k}, t_{k+1}\right)
$$

and $\mathcal{L}\left[e^{2 \alpha t} V_{e}(t)\right]=e^{2 \alpha t}\left[2 \alpha V_{e}(t)+\mathcal{L} V_{e}(t)\right], \alpha>0$, then $(21)$ implies

$$
\begin{aligned}
& \mathbb{E}\left\{V_{e}(t)\right\} \leq \mathbb{E}\left\{e^{-2 \alpha\left(t-t_{k}\right)} V_{e}\left(t_{k}\right)\right. \\
& \left.\quad+\sum_{i=1}^{2}\left(1-\beta_{i}\right) e_{i}^{T}\left(t_{k}\right)\left(Q_{i}-U_{i}\right) e_{i}\left(t_{k}\right)\right\}, t \in\left[t_{k}, t_{k+1}\right) .
\end{aligned}
$$

Therefore,

$$
\begin{aligned}
& \mathbb{E}\left\{V\left(t, x_{t}, \dot{x}_{t}\right)\right\} \leq \mathbb{E}\left\{e^{-2 \alpha\left(t-t_{k}\right)} V_{e}\left(t_{k}\right)\right. \\
&\left.-\sum_{i=1}^{2}\left(1-\beta_{i}\right) e_{i}^{T}\left(t_{k}\right) U_{i} e_{i}\left(t_{k}\right)\right\} \\
& \leq \mathbb{E}\left\{e^{-2 \alpha\left(t-t_{k}\right)} V_{e}\left(t_{k}\right)\right\}, t \in\left[t_{k}, t_{k+1}\right)
\end{aligned}
$$

Note that

$$
\begin{aligned}
V_{e}\left(t_{k+1}\right)= & \tilde{V}_{\mid t=t_{k+1}}+\sum_{i=1}^{2}\left(1-\beta_{i}\right) e_{i}^{T}\left(t_{k+1}\right) Q_{i} e_{i}\left(t_{k+1}\right) \\
& +\left(\tau_{M}-\eta_{m}\right) \int_{t_{k+1}-\eta_{k+1}}^{t_{k+1}} e^{2 \alpha\left(s-t_{k+1}\right)} \dot{x}^{T}(s) Q \dot{x}(s) d s
\end{aligned}
$$

and

$$
\begin{aligned}
& \mathbb{E}\left\{e_{i}^{T}\left(t_{k+1}\right) Q_{i} e_{i}\left(t_{k+1}\right)\right\} \\
& =\mathbb{E}\left\{\mid \sqrt{Q_{i}}\left[\left(1-\pi_{\left\{u_{k}=u^{i}\right\}}\right) e_{i}\left(t_{k}\right)\right.\right. \\
& \left.\left.\quad+C_{i} x\left(t_{k}-\eta_{k}\right)-C_{i} x\left(t_{k+1}-\eta_{k+1}\right)\right]\left.\right|^{2}\right\} \\
& =\mathbb{E}\left\{\left(1-\pi_{\left.\left\{u_{k}=u^{i}\right\}\right)^{2} e_{i}^{T}\left(t_{k}\right) Q_{i} e_{i}\left(t_{k}\right)}\right.\right. \\
& \quad+2\left(1-\pi_{\left\{u_{k}=u^{i}\right\}}\right) e_{i}^{T}\left(t_{k}\right) Q_{i}\left[C_{i} x\left(t_{k}-\eta_{k}\right)-C_{i} x\left(t_{k+1}-\eta_{k+1}\right)\right] \\
& \left.\quad+\left|\sqrt{Q_{i}}\left[C_{i} x\left(t_{k}-\eta_{k}\right)-C_{i} x\left(t_{k+1}-\eta_{k+1}\right)\right]\right|^{2}\right\} \\
& =\mathbb{E}\left\{\left(1-\beta_{i}\right) e_{i}^{T}\left(t_{k}\right) Q_{i} e_{i}\left(t_{k}\right)\right. \\
& \quad+2\left(1-\beta_{i}\right) e_{i}^{T}\left(t_{k}\right) Q_{i}\left[C_{i} x\left(t_{k}-\eta_{k}\right)-C_{i} x\left(t_{k+1}-\eta_{k+1}\right)\right] \\
& \left.\quad+\left|\sqrt{Q_{i}}\left[C_{i} x\left(t_{k}-\eta_{k}\right)-C_{i} x\left(t_{k+1}-\eta_{k+1}\right)\right]\right|^{2}\right\}, i=1,2 .
\end{aligned}
$$

Taking (22) with $i=1$ and (19) into account, we obtain

$$
\begin{aligned}
\mathbb{E}\{ & \left.V_{e}\left(t_{k+1}\right)-V_{e}\left(t_{k+1}^{-}\right)+\sum_{i=1}^{2}\left(1-\beta_{i}\right) e_{i}^{T}\left(t_{k}\right)\left(Q_{i}-U_{i}\right) e_{i}\left(t_{k}\right)\right\} \\
\leq & \mathbb{E}\left\{\sum_{i=1}^{2}\left(1-\beta_{i}\right) e_{i}^{T}\left(t_{k+1}\right) Q_{i} e_{i}\left(t_{k+1}\right)-\sum_{i=1}^{2}\left(1-\beta_{i}\right) e_{i}^{T}\left(t_{k}\right) Q_{i} e_{i}\left(t_{k}\right)\right. \\
& +\sum_{i=1}^{2}\left(1-\beta_{i}\right) e_{i}^{T}\left(t_{k}\right)\left(Q_{i}-U_{i}\right) e_{i}\left(t_{k}\right) \\
& \left.-e^{-2 \alpha \tau_{M}}\left|\sqrt{Q}\left[x\left(t_{k}-\eta_{k}\right)-x\left(t_{k+1}-\eta_{k+1}\right)\right]\right|^{2}\right\} \\
= & \mathbb{E}\left\{\sum_{i=1}^{2}\left(1-\beta_{i}\right) e_{i}^{T}\left(t_{k+1}\right) Q_{i} e_{i}\left(t_{k+1}\right)-\sum_{i=1}^{2}\left(1-\beta_{i}\right) e_{i}^{T}\left(t_{k}\right) U_{i} e_{i}\left(t_{k}\right)\right. \\
& \left.-e^{-2 \alpha \tau_{M}}\left|\sqrt{Q}\left[x\left(t_{k}-\eta_{k}\right)-x\left(t_{k+1}-\eta_{k+1}\right)\right]\right|^{2}\right\}
\end{aligned}
$$

$$
\begin{aligned}
= & \mathbb{E}\left\{\sum _ { i = 1 } ^ { 2 } \left\{\left(1-\beta_{i}\right) e_{i}^{T}\left(t_{k}\right)\left[\left(1-\beta_{i}\right) Q_{i}-U_{i}\right] e_{i}\left(t_{k}\right)\right.\right. \\
& +2\left(1-\beta_{i}\right)^{2} e_{i}^{T}\left(t_{k}\right) Q_{i}\left[C_{i} x\left(t_{k}-\eta_{k}\right)-C_{i} x\left(t_{k+1}-\eta_{k+1}\right)\right] \\
& \left.+\left(1-\beta_{i}\right)\left|\sqrt{Q_{i}}\left[C_{i} x\left(t_{k}-\eta_{k}\right)-C_{i} x\left(t_{k+1}-\eta_{k+1}\right)\right]\right|^{2}\right\} \\
& \left.-e^{-2 \alpha \tau_{M}}\left|\sqrt{Q}\left[x\left(t_{k}-\eta_{k}\right)-x\left(t_{k+1}-\eta_{k+1}\right)\right]\right|^{2}\right\} \\
= & \mathbb{E}\left\{\zeta(t)^{T} \Omega \zeta(t)\right\} \leq 0,
\end{aligned}
$$

where $\zeta(t)=\operatorname{col}\left\{x\left(t_{k}-\eta_{k}\right)-x\left(t_{k+1}-\eta_{k+1}\right), e_{1}\left(t_{k}\right), e_{2}\left(t_{k}\right)\right\}$ and $\Omega$ is given by $(22)$.

Therefore, (23) is valid and, together with (25) for $t=$ $t_{k+1}^{-}$, implies

$$
\begin{aligned}
\mathbb{E}\left\{V_{e}\left(t_{k+1}\right)\right\} & \leq e^{-2 \alpha\left(t_{k+1}-t_{k}\right)} \mathbb{E}\left\{V_{e}\left(t_{k}\right)\right\} \\
& \leq e^{-2 \alpha\left(t_{k+1}-t_{k-1}\right)} \mathbb{E}\left\{V_{e}\left(t_{k-1}\right)\right\} \\
& \leq e^{-2 \alpha\left(t_{k+1}-t_{0}\right)} \mathbb{E}\left\{V_{e}\left(t_{0}\right)\right\}
\end{aligned}
$$

The latter inequality, with $k+1$ replaced by $k$ and (26) give (24). The inequality (24) implies exponential meansquare stability of (13)-(14) with respect to $x$ because $\lambda_{\min }(P)|x(t)|^{2} \leq V\left(t, x_{t}, \dot{x}_{t}\right), V\left(t_{0}, x_{t_{0}}, \dot{x}_{t_{0}}\right) \leq v\left\|x_{t_{0}}\right\|_{W}^{2}$ for some scalar $v>0$.

Based on the above lemma, we derive LMI conditions for the exponential mean-square stability with respect to $x$ :

Theorem 1. Given $0 \leq \eta_{m}<\tau_{M}, \alpha>0, \beta_{i}>0, i=0,1,2$, $\sum_{i=0}^{2} \beta_{i}=1$ and $K_{1}, K_{2}$. Suppose there exist $n \times n$ matrices $P>0, Q>0, S_{j}>0, R_{j}>0, j=0,1, S_{12}$ and matrices $Q_{l}>0, U_{l}>0, l=1,2$ such that $(22)$ and

$$
\begin{aligned}
& {\left[\begin{array}{cc}
R_{1} & S_{12} \\
* & R_{1}
\end{array}\right] \geq 0,} \\
& {\left[\begin{array}{cc}
\Sigma_{11} & \Sigma_{12}^{T} \\
* & \Sigma_{22}
\end{array}\right]<0,}
\end{aligned}
$$

are feasible, where

$$
\begin{aligned}
& \Sigma_{11}=\left[\begin{array}{cccccc}
\varphi_{11} & R_{0} e^{-2 \alpha \eta_{m}} & P A_{1} & 0 & \varphi_{51} & \varphi_{52} \\
* & \varphi_{22} & \varphi_{23} & S_{12} e^{-2 \alpha \tau_{M}} & 0 & 0 \\
* & * & \varphi_{33} & \varphi_{23} & 0 & 0 \\
* & * & * & \varphi_{44} & 0 & 0 \\
* & * & * & * & \psi_{1} & 0 \\
* & * & * & * & * & \psi_{2}
\end{array}\right], \\
& \Sigma_{12}=\left[\begin{array}{cccccc}
H^{T} A & 0 & H^{T} A_{1} & 0 & \beta_{2} H^{T} B_{1} & \beta_{1} H^{T} B_{2} \\
0 & 0 & 0 & 0 & -\beta_{1}\left(1-\beta_{1}\right) H^{T} B_{1} & 0 \\
0 & 0 & 0 & 0 & 0 & -\beta_{2}\left(1-\beta_{2}\right) H^{T} B_{2}
\end{array}\right] \text {, } \\
& \Sigma_{22}=\operatorname{diag}\left\{-H,-\beta_{1}\left(1-\beta_{1}\right) H,-\beta_{2}\left(1-\beta_{2}\right) H\right\} \text {, } \\
& \varphi_{11}=P A+A^{T} P+S_{0}-R_{0} e^{-2 \alpha \eta_{m}}+2 \alpha P \text {, } \\
& \varphi_{22}=\left(-S_{0}+S_{1}-R_{0}\right) e^{-2 \alpha \eta_{m}}-R_{1} e^{-2 \alpha \tau_{M}} \text {, } \\
& \varphi_{23}=\left(R_{1}-S_{12}\right) e^{-2 \alpha \tau_{M}} \\
& \varphi_{33}=\left(-2 R_{1}+S_{12}+S_{12}^{T}\right) e^{-2 \alpha \tau_{M}}, \\
& \varphi_{44}=-\left(S_{1}+R_{1}\right) e^{-2 \alpha \tau_{M}} \text {, } \\
& \varphi_{5 i}=\left(\beta_{0}+\beta_{3-i}\right) P B_{i} \text {, } \\
& \psi_{i}=-\frac{1-\beta_{i}}{\tau_{M}-\eta_{m}}\left(Q_{i}-U_{i}\right)+2 \alpha \beta_{3-i} Q_{i}, \\
& H=\eta_{m}^{2} R_{0}+\left(\tau_{M}-\eta_{m}\right)^{2} R_{1}+\left(\tau_{M}-\eta_{m}\right) Q, i=1,2 .
\end{aligned}
$$

Then, the solutions of (13)-(14) satisfy the bound (24). Hence, the closed-loop system with initial condition $x_{t_{0}}, e\left(t_{0}\right)$ is exponentially mean-square stable with respect to $x$. 
Proof: Consider $t \in\left[t_{k}, t_{k+1}\right), k \in \mathbb{Z}$. Using the infinitesimal operator (20), we obtain

$$
\begin{aligned}
& \mathcal{L} V_{e}(t)+2 \alpha V_{e}(t)-\sum_{i=1}^{2} \frac{1-\beta_{i}}{\tau_{M}-\eta_{m}} e_{i}^{T}(t)\left(Q_{i}-U_{i}\right) e_{i}(t) \\
& \leq 2 x^{T}(t) P\left[A x(t)+A_{1} x(t-\tau(t))+\sum_{i=1}^{2}\left(1-\beta_{i}\right) B_{i} e_{i}(t)\right] \\
& +\sum_{i=1}^{2}\left(1-\beta_{i}\right) e_{i}^{T}(t)\left[2 \alpha Q_{i}-\frac{1}{\tau_{M}-\eta_{m}}\left(Q_{i}-U_{i}\right)\right] e_{i}(t) \\
& -x^{T}\left(t-\eta_{m}\right)\left[S_{0}-S_{1}\right] e^{-2 \alpha \eta_{m}} x\left(t-\eta_{m}\right) \\
& -x^{T}\left(t-\tau_{M}\right) S_{1} e^{-2 \alpha \tau_{M}} x\left(t-\tau_{M}\right)+\dot{x}^{T}(t) H \dot{x}(t) \\
& +x^{T}(t)\left(S_{0}+2 \alpha P\right) x(t)-\eta_{m} e^{-2 \alpha \eta_{m}} \int_{t-\eta_{m}}^{t} \dot{x}^{T}(s) R_{0} \dot{x}(s) d s \\
& -\left(\tau_{M}-\eta_{m}\right) e^{-2 \alpha \tau_{M}} \int_{t-\tau_{M}}^{t-\eta_{m}} \dot{x}^{T}(s) R_{1} \dot{x}(s) d s .
\end{aligned}
$$

By Jensen's inequality (Gu et al. [2003])

$$
\begin{aligned}
& \eta_{m} \int_{t-\eta_{m}}^{t} \dot{x}^{T}(s) R_{0} \dot{x}(s) d s \\
& \geq \int_{t-\eta_{m}}^{t} \dot{x}^{T}(s) d s R_{0} \int_{t-\eta_{m}}^{t} \dot{x}(s) d s \\
& =\left[x(t)-x\left(t-\eta_{m}\right)\right]^{T} R_{0}\left[x(t)-x\left(t-\eta_{m}\right)\right]
\end{aligned}
$$

and

$$
\begin{aligned}
& -\left(\tau_{M}-\eta_{m}\right) \int_{t-\tau_{M}}^{t-\eta_{m}} \dot{x}^{T}(s) R_{1} \dot{x}(s) d s \\
& =-\left(\tau_{M}-\eta_{m}\right) \int_{t-\tau(t)}^{t-\eta_{m}} \dot{x}^{T}(s) R_{1} \dot{x}(s) d s \\
& -\left(\tau_{M}-\eta_{m}\right) \int_{t-\tau(t)}^{t-\tau_{M}} \dot{x}^{T}(s) R_{1} \dot{x}(s) d s \\
& \leq-\frac{\tau_{M}-\eta_{m}}{\tau(t)-\eta_{m}} f_{1}^{T}(t) R_{1} f_{1}(t)-\frac{\tau_{M}-\eta_{m}}{\tau_{M}-\tau(t)} f_{2}^{T}(t) R_{1} f_{2}(t) \\
& \leq-\left[\begin{array}{c}
f_{1}(t) \\
f_{2}(t)
\end{array}\right]^{T}\left[\begin{array}{cc}
R_{1} & S_{12} \\
* & R_{1}
\end{array}\right]\left[\begin{array}{l}
f_{1}(t) \\
f_{2}(t)
\end{array}\right],
\end{aligned}
$$

where $f_{1}(t)=x\left(t-\eta_{m}\right)-x(t-\tau(t)), f_{2}(t)=x(t-\tau(t))-$ $x\left(t-\tau_{M}\right)$. The latter inequality holds if (28) is feasible (Park et al. [2011]).

Define $\xi(t)=\operatorname{col}\left\{x(t), x\left(t-\eta_{m}\right), x(t-\tau(t)), x(t-\right.$ $\left.\left.\tau_{M}\right), e_{1}(t), e_{2}(t)\right\}$. It can be shown from (13) that

$$
\begin{aligned}
& \dot{x}(t)=\Xi \xi(t)+\sum_{i=1}^{2}\left[\pi_{\left\{u_{k}=u^{i}\right\}}-\beta_{i}\right] \Xi_{i} \xi(t), \\
& \dot{x}^{T}(t) H \dot{x}(t)=\xi^{T}(t) \Xi^{T} H \Xi \xi(t) \\
& \quad+2 \sum_{i=1}^{2}\left[\pi_{\left\{u_{k}=u^{i}\right\}}-\beta_{i}\right] \xi^{T}(t) \Xi^{T} H \Xi_{i} \xi(t) \\
& \quad+2\left[\pi_{\left\{u_{k}=u^{1}\right\}}-\beta_{1}\right]\left[\pi_{\left\{u_{k}=u^{2}\right\}}-\beta_{2}\right] \xi^{T}(t) \Xi_{1}^{T} H \Xi_{2} \xi(t) \\
& \quad+\sum_{i=1}^{2}\left[\pi_{\left\{u_{k}=u^{i}\right\}}-\beta_{i}\right]^{2} \xi^{T}(t) \Xi_{i}^{T} H \Xi_{i} \xi(t),
\end{aligned}
$$

where

$$
\begin{aligned}
& \Xi=\left[\begin{array}{lllll}
A & 0 & A_{1} & 0 & \left(\beta_{0}+\beta_{2}\right) B_{1} \\
\Xi & \left(\beta_{0}+\beta_{1}\right) B_{2}
\end{array}\right], \\
& \Xi_{1}=\left[\begin{array}{llllll}
0 & 0 & 0 & 0 & -B_{1} & 0
\end{array}\right], \\
& \Xi_{2}=\left[\begin{array}{llllll}
0 & 0 & 0 & 0 & 0 & -B_{2}
\end{array}\right] .
\end{aligned}
$$

Substituting (32)-(34) into (31) and taking expectation on both sides of (31), we get

$$
\begin{aligned}
& \mathbb{E}\left\{\mathcal{L} V_{e}(t)+2 \alpha V_{e}(t)-\sum_{i=1}^{2} \frac{1-\beta_{i}}{\tau_{M}-\eta_{m}} e_{i}^{T}(t)\left(Q_{i}-U_{i}\right) e_{i}(t)\right\} \\
& \leq \mathbb{E}\left\{\xi^{T}(t)\left[\Sigma_{11}+\Xi^{T} H \Xi+\sum_{i=1}^{2} \beta_{i}\left(1-\beta_{i}\right) \Xi_{i}^{T} H \Xi_{i}\right] \xi(t)\right\},
\end{aligned}
$$

where the notations are given by (30). Further, by application of Schur complements, it follows that (28) and (29) guarantee $(21)$ for $t \in\left[t_{k}, t_{k+1}\right), \xi(t) \neq 0$. Thus by Lemma 1, LMIs (22), (28) and (29) imply (24).

Remark 5. When $\beta_{0}=0, \beta_{i}=\frac{1}{2}, i=1,2$, the stabilization of NCSs under the RR protocol can be included in this case. Compare the number of scalar decision variables in the LMIs under the RR protocol in Liu et al. [2012b] and under the stochastic protocol in Theorem 1. The LMIs (two of $6 n \times 6 n$ and two of $3 n \times 3 n$ ) under the RR protocol have $8.5 n^{2}+2.5 n$ variables, which is bigger than the number $4.25 n^{2}+3.5 n$ of variables (for $y_{i}\left(s_{k}\right) \in \mathcal{R}^{n / 2}(i=$ $1,2)$ ) in the LMIs (one of $8 n \times 8 n$ and two of $2 n \times 2 n$ ) under the stochastic protocol in Theorem 1. Under the TOD protocol in Liu et al. [2012a], the number of variables is $4.25 n^{2}+3.5 n$, but in two of $5.5 n \times 5.5 n$ LMIs and one of $2 n \times 2 n$ LMI.

Remark 6 . The LMIs of Theorem 1 are affine in the system matrices. Therefore, in the case of system matrices from the uncertain time-varying polytope

$$
\begin{aligned}
& \Theta=\sum_{j=1}^{M} g_{j}(t) \Theta_{j}, \quad 0 \leq g_{j}(t) \leq 1, \\
& \sum_{j=1}^{M} g_{j}(t)=1, \quad \Theta_{j}=\left[A^{(j)} B^{(j)}\right],
\end{aligned}
$$

one have to solve these LMIs simultaneously for all the $M$ vertices $\Theta_{j}$, applying the same decision matrices.

\section{EXAMPLES}

\subsection{Example 1: Cart-pendulum system}

Consider the following linearized model of the inverted pendulum on a cart:

$$
\left[\begin{array}{c}
\dot{x} \\
\ddot{x} \\
\dot{\theta} \\
\ddot{\theta}
\end{array}\right]=\left[\begin{array}{cccc}
0 & 1 & 0 & 0 \\
0 & 0 & \frac{-m g}{M} & 0 \\
0 & 0 & 0 & 1 \\
0 & 0 & \frac{(M+m) g}{M l} & 0
\end{array}\right]\left[\begin{array}{c}
x \\
\dot{x} \\
\theta \\
\dot{\theta}
\end{array}\right]+\left[\begin{array}{c}
0 \\
\frac{a}{M} \\
0 \\
\frac{-a}{M l}
\end{array}\right] u
$$

with $M=3.9249 \mathrm{~kg}, m=0.2047 \mathrm{~kg}, l=0.2302 \mathrm{~m}, g=$ $9.81 \mathrm{~N} / \mathrm{kg}, a=25.3 \mathrm{~N} / \mathrm{V}$. In the model, $x$ and $\theta$ represent cart position coordinate and pendulum angle from vertical, respectively.

The pendulum can be stabilized by a state feedback $u(t)=$ $K\left[\begin{array}{llll}x & \dot{x} & \theta & \dot{\theta}\end{array}\right]^{T}$ with the gain

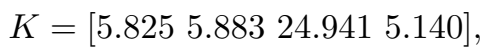


which leads to the closed-loop system eigenvalues $\{-100,-2+$ $2 i,-2-2 i,-2\}$. Suppose the variables $\theta, \dot{\theta}$ and $x, \dot{x}$ are not accessible simultaneously. We consider

$$
C_{1}=\left[\begin{array}{llll}
1 & 0 & 0 & 0 \\
0 & 1 & 0 & 0
\end{array}\right], C_{2}=\left[\begin{array}{llll}
0 & 0 & 1 & 0 \\
0 & 0 & 0 & 1
\end{array}\right] .
$$

The applied control is obtained from the blocks of $K$ :

$$
K_{1}=\left[\begin{array}{ll}
5.825 & 5.883
\end{array}\right], K_{2}=\left[\begin{array}{ll}
24.941 & 5.140
\end{array}\right] \text {. }
$$

Assume that $\beta_{0}=0$, which means that the event of collision does not occur. Choose $\beta_{1}=0.3$. For the values of $\eta_{m}$ given in Table 1 , we apply Theorem 1 with $\alpha=0$ and find the maximum values of $\tau_{M}=M A T I+\eta_{M}$ that preserve the mean-square stability of the impulsive system (13)-(14) with respect to $x$ (see Table 1$)$. It can be found from Table 1 that, when $\eta_{m}>\frac{\tau_{M}}{2}\left(\eta_{m}=0.004,0.005\right)$, our method is feasible. Choosing $\eta_{m}=0.002$, by Theorem 1 with $\alpha=0$, we obtain the corresponding maximum values of $\tau_{M}$ shown in Fig. 2 for different $\beta_{1}$.

Table 1. Example 1: $\max$. value of $\tau_{M}=$ $M A T I+\eta_{M}$ for different $\eta_{m}$

\begin{tabular}{|c|c|c|c|c|c|c|}
\hline$\tau_{M} \backslash \eta_{m}$ & 0 & 0.001 & 0.002 & 0.003 & 0.004 & 0.005 \\
\hline \hline $\begin{array}{c}\text { Liu et al. }[2012 \mathrm{~b}] \\
\text { (RR) }\end{array}$ & 0.0040 & 0.0047 & 0.0054 & 0.0061 & 0.0068 & 0.0075 \\
\hline $\begin{array}{c}\text { Liu et al. }[2012 \mathrm{a}] \\
\text { (TOD) }\end{array}$ & 0.0042 & 0.0049 & 0.0056 & 0.0062 & 0.0069 & 0.0075 \\
\hline $\begin{array}{c}\text { Theorem } 1 \\
\left(\beta_{1}=0.3\right)\end{array}$ & 0.0032 & 0.0040 & 0.0047 & 0.0054 & 0.0062 & 0.0069 \\
\hline
\end{tabular}

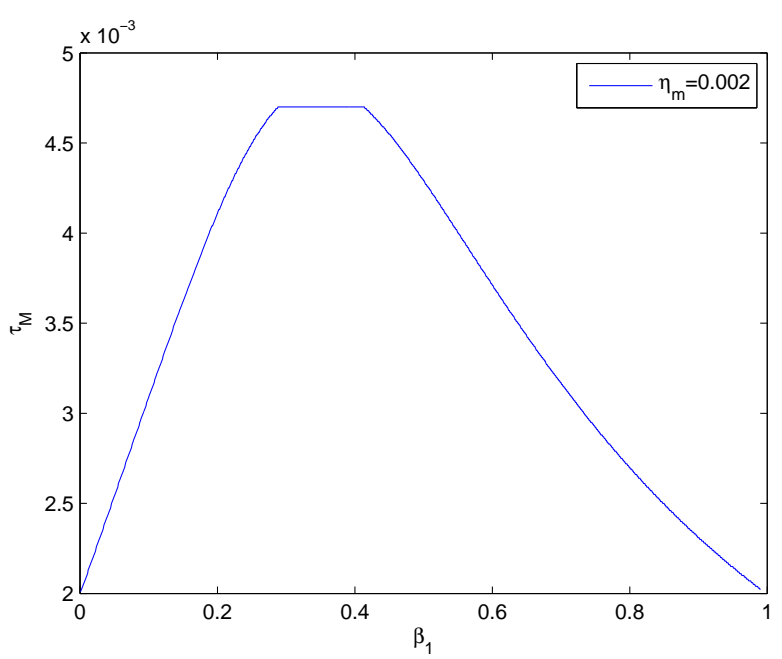

Fig. 2. Example 1: effect of $\beta_{1}$ on the $\tau_{M}$ for $\beta_{0}=0$ and $\eta_{m}=0.002$

Choosing $\eta_{m}=0.003, \tau_{M}=0.0035$, by applying Theorem 1 , we obtain the maximum values of the decay rate $\alpha$ shown in Fig. 3 for different $\beta_{1}$.

\subsection{Example 2: Batch reactor}

We illustrate the efficiency of the given conditions on a benchmark example of a batch reactor under the dynamic output feedback with $\eta_{k}=\eta_{k}^{s c}$ (Donkers et al. [2011], Heemels et al. [2010]), where

$$
A=\left[\begin{array}{cccc}
1.380 & -0.208 & 6.715 & -5.676 \\
-0.581 & -4.2902 & 0 & 0.675 \\
1.067 & 4.273 & -6.654 & 5.893 \\
0.048 & 4.273 & 1.343 & -2.104
\end{array}\right]
$$

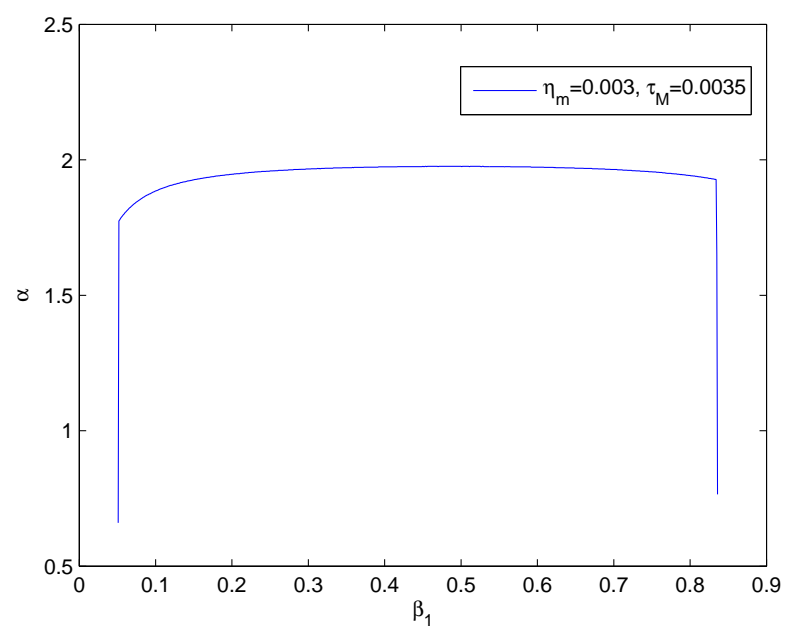

Fig. 3. Example 1: effect of $\beta_{1}$ on the decay rate $\alpha$ for $\beta_{0}=0, \eta_{m}=0.003$ and $\tau_{M}=0.0035$

$$
\begin{gathered}
B=\left[\begin{array}{cc}
0 & 0 \\
5.679 & 0 \\
1.136 & -3.146 \\
1.136 & 0
\end{array}\right], C=\left[\frac{C_{1}}{C_{2}}\right]=\left[\begin{array}{llll}
1 & 0 & 1 & -1 \\
\hline 0 & 1 & 0 & 0
\end{array}\right], \\
{\left[\begin{array}{c|c}
A_{c} & B_{c} \\
\hline C_{c} & D_{c}
\end{array}\right]=\left[\begin{array}{cc|cc}
0 & 0 & 0 & 1 \\
0 & 0 & 1 & 0 \\
\hline-2 & 0 & 0 & -2 \\
0 & 8 & 5 & 0
\end{array}\right]}
\end{gathered}
$$

Assume that $\beta_{0}=0$, which means that the event of collision does not occur. Let $\beta_{1}=0.6$. For the values of $\eta_{m}$ given in Table 2 , we apply Theorem 1 with $\alpha=0$ and find the maximum values of $\tau_{M}=M A T I+\eta_{M}$ that preserve the mean-square stability of the impulsive system (13)-(14) with respect to $x$ (see Table 2). From Table 2 it is seen that the results of our method essentially improve the results in Heemels et al. [2010] in terms of both TOD and RR protocols. Moreover, we can find that the introduced stochastic protocol stabilizes the system for smaller $\tau_{M}$ than the RR and TOD, which have been developed based on the time-delay approach in Liu et al. [2012b] and Liu et al. [2012a], respectively. When $\eta_{m}>$ $\frac{\tau_{M}}{2}\left(\eta_{m}=0.03,0.04\right)$, our method is applicable. Choosing $\eta_{m}=0.02$, by Theorem 1 with $\alpha=0$, we obtain the corresponding maximum values of $\tau_{M}$ shown in Fig. 4 for different $\beta_{1}$.

Table 2. Example: max. value of $\tau_{M}=$ $M A T I+\eta_{M}$ for different $\eta_{m}$

\begin{tabular}{|c|c|c|c|c|c|}
\hline$\tau_{M} \backslash \eta_{m}$ & 0 & 0.004 & 0.02 & 0.03 & 0.04 \\
\hline \hline $\begin{array}{c}\text { Heemels et al. [2010] } \\
\left(\eta_{M}=0.004, \text { TOD) }\right.\end{array}$ & 0.0108 & 0.0133 & - & - & - \\
\hline $\begin{array}{c}\text { Heemels et al. [2010] } \\
\left(\eta_{M}=0.004, \mathrm{RR}\right)\end{array}$ & 0.0088 & 0.0088 & - & - & - \\
\hline $\begin{array}{c}\text { Donkers et al. [2011] } \\
\left(\eta_{M}=0.03, \text { TOD) }\right.\end{array}$ & 0.069 & 0.069 & 0.069 & 0.069 & - \\
\hline $\begin{array}{c}\text { Donkers et al. [2011] } \\
\left(\eta_{M}=0.03, \mathrm{RR}\right)\end{array}$ & 0.068 & 0.068 & 0.068 & 0.068 & - \\
\hline Liu et al. [2012a](TOD) & 0.035 & 0.037 & 0.047 & 0.053 & 0.059 \\
\hline Liu et al. [2012b] (RR) & 0.042 & 0.044 & 0.053 & 0.058 & 0.063 \\
\hline Theorem 1 $\left(\beta_{1}=0.6\right)$ & 0.025 & 0.028 & 0.041 & 0.050 & 0.058 \\
\hline
\end{tabular}




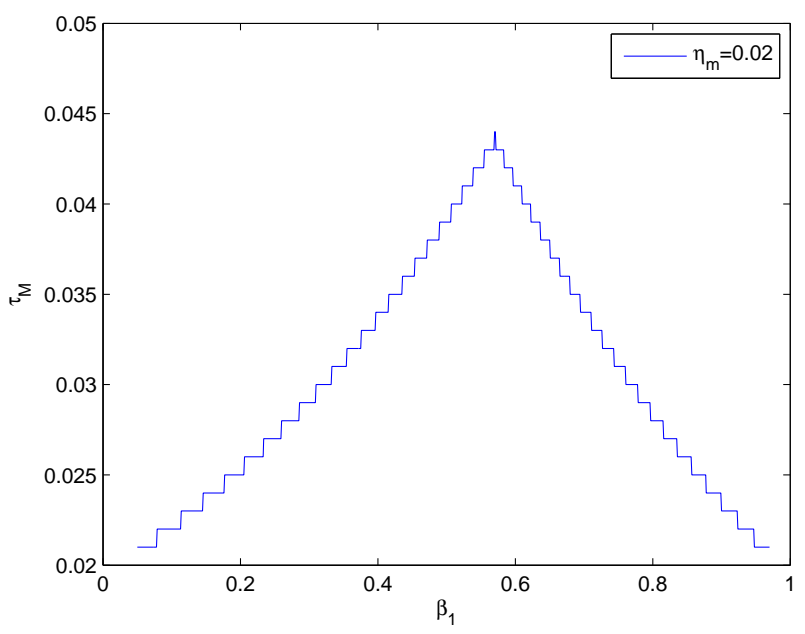

Fig. 4. Example 2: effect of $\beta_{1}$ on the $\tau_{M}$ for $\beta_{0}=0$ and $\eta_{m}=0.02$

Choosing $\eta_{m}=0.02, \tau_{M}=0.025$, by applying Theorem 1 , we obtain the maximum values of the decay rate $\alpha$ shown in Fig. 5 for different $\beta_{1}$.

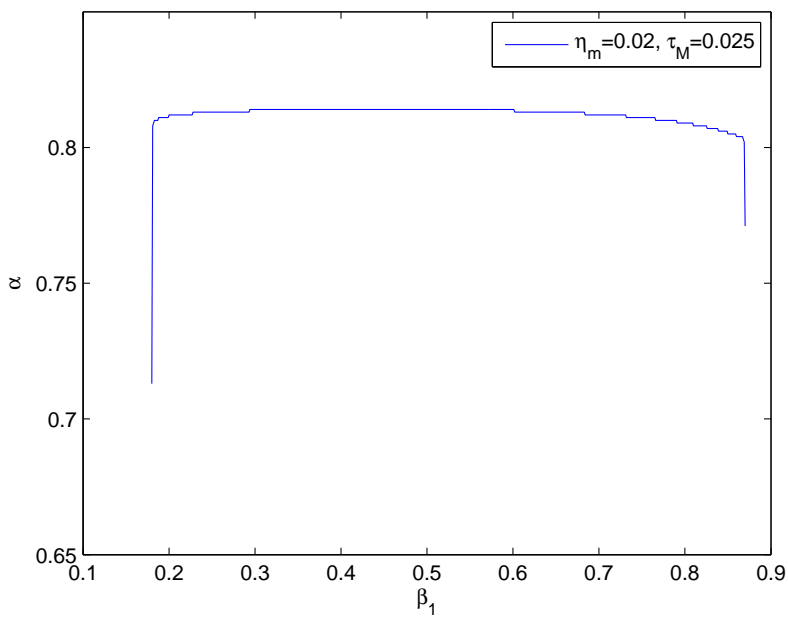

Fig. 5. Example 2: effect of $\beta_{1}$ on the decay rate $\alpha$ for $\beta_{0}=0, \eta_{m}=0.02$ and $\tau_{M}=0.025$

Remark 7. Simulations in both examples above show that our stochastic protocol is conservative compared to the TOD protocol in Liu et al. [2012a] and RR protocol in Liu et al. [2012b]. However, we cannot immediately conclude that among the scheduling protocols based on time-delay approach, TOD and RR are superior to stochastic protocol. The results in this paper could be further improved in terms of other stochastic protocols.

\section{CONCLUSIONS}

In this paper, a time-delay approach was developed for the stabilization of NCSs under a stochastic protocol, variable delays and variable sampling intervals. Differently from Liu et al. [2012b] and Liu et al. [2012a] for deterministic protocols, collisions can occur with a certain probability under the stochastic protocol. Moreover, the resulting LMIs are simpler. The closed-loop system was modeled as an impulsive time-delay system with stochastic parameters. By developing appropriate Lyapunov-Krasovskiibased methods, the exponential mean-square stability conditions for the delayed impulsive system were derived in terms of LMIs.

\section{REFERENCES}

P. Antsaklis and J. Baillieul. Special issue on technology of networked control systems. Proceedings of the IEEE, 95(1):5-8, 2007.

M. Donkers, W. Heemels, N. van de Wouw, and L. Hetel. Stability analysis of networked control systems using a switched linear systems approach. IEEE Transactions on Automatic control, 56(9):2101-2115, 2011.

M.C.F. Donkers, W. Heemels, D. Bernardini, A. Bemporad, and V. Shneer. Stability analysis of stochastic networked control systems. Automatica, 48(5):917-925, 2012.

E. Fridman. A refined input delay approach to sampleddata control. Automatica, 46(2):421-427, 2010.

E. Fridman, A. Seuret, and J.P. Richard. Robust sampleddata stabilization of linear systems: an input delay approach. Automatica, 40(8):1441-1446, 2004.

H. Fujioka. Stability analysis of systems with aperiodic sample-and-hold devices. Automatica, 45(3):771-775, 2009.

H. Gao and T. Chen. Network-based $H_{\infty}$ output tracking control. IEEE Transactions on Automatic control, 53 (3):655-667, 2008.

H. Gao, J. Wu, and P. Shi. Robust sampled-data $H_{\infty}$ control with stochastic sampling. Automatica, 45(7): 1729-1736, 2009.

K. Gu, V. Kharitonov, and J. Chen. Stability of TimeDelay Systems. Birkhäuser, Boston, 2003.

W.P.M.H. Heemels, A.R. Teel, N. van de Wouw, and D. Nesic. Networked control systems with communication constraints: tradeoffs between transmission intervals, delays and performance. IEEE Transactions on Automatic control, 55(8):1781-1796, 2010.

D. Huang and S.K. Nguang. State feedback control of uncertain networked control systems with random time delays. IEEE Transactions on Automatic control, 53(3): 829-834, 2008.

K. Liu, E. Fridman, and L. Hetel. Network-based control via a novel analysis of hybrid systems with time-varying delays. In Proceedings of the 51th IEEE Conference on Decision and Control, Hawaii, USA, December 2012a.

K. Liu, E. Fridman, and L. Hetel. Stability and $L_{2}$-gain analysis of networked control systems under RoundRobin scheduling: a time-delay approach. Systems \& Control Letters, 61(5):666-675, 2012b.

P. Naghshtabrizi, J.P. Hespanha, and A.R. Teel. Stability of delay impulsive systems with application to networked control systems. Transactions of the Institute of Measurement and Control, 32(5):511-528, 2010.

D. Nesic and D. Liberzon. A unified framework for design and analysis of networked and quantized control systems. IEEE Transactions on Automatic control, 54 (4):732-747, 2009 .

J. Nilsson. Real-time control systems with delays. $\mathrm{PhD}$ thesis, Lund Institute of Technology, 1998.

P.G. Park, J.W. Ko, and C. Jeong. Reciprocally convex approach to stability of systems with time-varying de- 
lays. Automatica, 47(1):235-238, 2011.

M. Tabbara and D. Nesic. Input-output stability of networked control systems with stochastic protocols and channels. IEEE Transactions on Automatic control, 53 (5):1160-1175, 2008.

Z. Wang, F. Yang, D.W.C. Ho, and X. Liu. Robust $H_{\infty}$ filtering for stochastic time-delay systems with missing measurements. IEEE Transactions on Signal Processing, 54(7):2579-2587, 2006.

Z. Wang, F. Yang, D.W.C. Ho, and X. Liu. Robust $H_{\infty}$ control for networked systems with random packet losses. IEEE Transactions on Systems, Man, and Cybernetics-Part B, 37(4):916-924, 2007.

D. Yue, E. Tian, Y Zhang, and C. Peng. Delaydistribution-dependent robust stability of uncertain systems with time-varying delay. International Journal of Robust and Nonlinear Control, 19(4):377-393, 2009. 